\title{
Spontaneous rupture of the quadriceps tendon in patients on chronic haemodialysis
}

\author{
M. LOTEM, M. D. ROBSON, AND J. B. ROSENFELD \\ From the Department of Orthopaedics and Traumatology and the Renal Unit, Beilinson Medical Center, \\ Tel Aviv University Medical School, Petah Tiqva, Israel
}

With the advent of chronic haemodialysis the rheumatologist and orthopaedic surgeon have been faced with many new and challenging problems. Dialytic bone disease, avascular necrosis of the femoral head (Baily, Griffitts, Mocelin, Gundy, Hampers, and Merrill, 1972) and extensive soft tissue calcifications constitute some of the unsolved problems of chronic haemodialysis.

We present two cases of spontaneous rupture of the quadriceps tendon, a complication which to our knowledge has not been previously described as associated with haemodialysis.

\section{Case reports}

\section{Case 1}

A 32-year-old male 10 years previously had been diagnosed as suffering from chronic pyelonephritis and vascular hypertension. For the past 2 years he had been treated by chronic haemodialysis. He showed signs of hyperparathyroidism such as $x$-ray changes in the phalanges and long bones, a blood calcium of $10.6 \mathrm{mg} / 100 \mathrm{ml}$, phosphorus $8.7 \mathrm{mg} / 100 \mathrm{ml}$, and alkaline phosphatase 6 Bessey Lowry units. On the morning after a haemodialysis, while the patient was walking down the stairs of the unit, his knees gave way suddenly without apparent reason. He was unable to rise or to extend the left knee. The right knee was also painful. Haemarthrosis of both knees was found, with maximal tenderness over the upper pole of the patellae. The $x$-ray showed avulsion of small flakes of bone from the upper poles of the patellae. A diagnosis of spontaneous rupture of quadriceps tendons was madecomplete on the left side and partial on the right. The left knee was explored. The tendon was found to be frayed and the lesion resembled a degenerative tear. The torn tendon was sutured back to the patella and a plaster cylinder applied for 6 weeks. The right knee was treated by pressure bandage. Recovery was complete on both sides.

\section{Case 2}

A male patient had been suffering from chronic glomerulonephritis from the age of 7 . Intermittent haemodialysis was started at the age of 16.2 years later he fell down without apparent reason. The left knee was found to be tender, and a small flake of bone was found to be avulsed from the upper pole of the patella. A plaster cylinder was applied for 4 weeks. A similar episode occurred 4 months later on the same side and was treated similarly. Recovery was complete.

\section{Discussion}

Murphy and McPhee (1965) described a case of simultaneous spontaneous rupture of the quadriceps, patella, and triceps tendons in a 39-year-old man who suffered from renal insufficiency and chronic acidosis A biopsy of the tendon showed many fibres which stained with elastic tissue stains. He attributed tho tendon weakness to a connective tissue elastosis associated with chronic acidosis.

Connective tissue elastosis was found in elastic tissues of dermis, lung, aorta, cartilage, and bone in a series of patients suffering from chronic acidosis and was attributed to the acidosis (Finlayson, Smith, and Moore, 1964).

It is probable that the case of rupture of the quadriceps tendon in gout described by Levy, Seelenfreund, Maor, Fried, and Lurie (1971), where the rupture was explained by infiltration of the tendon with uric acid crystals, was in fact due to changes of connective tissue elastosis, since the patient was known to be in terminal renal failure and had a severe metabolic acidosis.

Spontaneous rupture of tendons is the result of a degenerative process and is usually found in patients in the sixth decade. It is possible that the degeneration of the tendons observed in these two relatively young patients was accelerated by the uraemic environment

\section{Summary}

Two cases of spontaneous rupture of the quadriceps tendon in two young male patients on chronic haemodialysis are presented. It is suggested that the rupture was due to connective tissue elastosis resulting from chronic acidosis. 


\section{References}

Baily, G. L., Griffitts, H. J., Mocelin, A. J., Gundy, D. H., Hampers, C. L., AND MerRill, J. P. (1972) Trans. Amer. Soc. artif. Intern. Org., 18, 401 (Avascular necrosis of the femoral head in patients on chronic hemodialysis)

Finlayson, G. R., Smith, J. G., AND Moore, M. J. (1964) J. Amer. med. Ass., 187, 659 (Effects of chronic acidosis on connective tissue)

Levy, M., Seelenfreund, M., MaOr, P., Fried, A., And Lurie, M. (1971) J. Bone Jt. Surg., 53-B, 510 (Bilateral spontaneous and simultaneous rupture of the quadriceps tendon in gout)

MurPHY, K. J., AND MCPheE, I. (1965) Ibid., 47-A, 1253 (Tears of major tendons in chronic acidosis with elastosis) 